\title{
Low Releasing Mitomycin C Molecule Encapsulated with Chitosan Nanoparticles for Intravesical Installation
}

\author{
Doğa Kavaz $^{1,2 *}$, Feyza Kirac ${ }^{3}$, Mustafa Kirac ${ }^{4}$, Ashok Vaseashta ${ }^{5,6}$ \\ ${ }^{1}$ Bioengineering Department, Cyprus International University, Nicosia, Northern Cyprus \\ ${ }^{2}$ Biotechnology Research Center, Cyprus International University, Nicosia, Northern Cyprus \\ ${ }^{3}$ Department of Chemistry, Hacettepe University, Ankara, Turkey \\ ${ }^{4}$ Department of Urology, Koru Ankara Hospital, Ankara, Turkey \\ ${ }^{5}$ International Clean Water Institute, Manassas, VA, USA \\ ${ }^{6}$ Office of Research, NJCU-A State University of New Jersey, Jersey City, NJ, USA \\ Email: *prof.vaseashta@ieee.org
}

How to cite this paper: Kavaz, D., Kirac, F., Kirac, M. and Vaseashta, A. (2017) Low Releasing Mitomycin C Molecule Encapsulated with Chitosan Nanoparticles for Intravesical Installation. Journal of Biomaterials and Nanobiotechnology, 8, 203-219. https://doi.org/10.4236/jbnb.2017.84014

Received: July 16, 2017

Accepted: August 29, 2017

Published: September 1, 2017

Copyright $\odot 2017$ by authors and Scientific Research Publishing Inc. This work is licensed under the Creative Commons Attribution International License (CC BY 4.0).

http://creativecommons.org/licenses/by/4.0/

\begin{abstract}
The aim of this investigation is preparation of Mitomycin-C encapsulated with chitosan nanoparticles synthesis using ionic gelation technique for intravesical controlled drug delivery systems. This study was conducted in vitro. Cumulative amount of drug released from the nanoparticles was calculated. Mitomycin- $\mathrm{C}$ release studies were examined for different $\mathrm{pH}$ values. During the drug loading and release studies, initial amount of drug was changed (i.e., 0.5, 1.25 and $2.5 \mathrm{mg}$ ) to get different release profiles and the release studies were repeated $(n=6)$. The loading efficiencies of Mitomycin-C with three different initial concentrations $0.5 \mathrm{mg} / \mathrm{ml}, 1.25 \mathrm{mg} / \mathrm{ml}$ and $2.5 \mathrm{mg} / \mathrm{ml}$ into chitosan nanoparticles were $54.5 \%, 47.1 \%$ and $36.4 \%$, respectively. For different $\mathrm{pH}$ values, the cumulative releases of Mitomycin-C from chitosan nanoparticles were $47 \%$ and $53 \%$ for $\mathrm{pH} 6.0$ and 7.4 , respectively $(p<0.01)$. For different drug doses, the cumulative releases of Mitomycin-C (MMC) from Chitosan nanoparticles were $44 \%, 53 \%$ and $65 \%$ for $0.5 \mathrm{mg} / \mathrm{mL}, 1.25 \mathrm{mg} / \mathrm{mL}$ and $2.5 \mathrm{mg} / \mathrm{mL}$ respectively $(p<0.01)$. The anticancer activity of Mitomycin-C loaded chitosan nanoparticles was measured in T24 bladder cancer cell line in vitro, and the results revealed that the $2.5 \mathrm{MMC}$ coated Chitosan nanoparticles had better tumor cells decline activity. From this investigation, we conclude that the drug encapsulated synthesized chitosan nanoparticles possess a high ability to be used as $\mathrm{pH}$ and dose responsive drug delivery system. This systematic investigation demonstrates a promising future for the intravesical installation in treatment of the superficial bladder cancer.
\end{abstract}




\section{Keywords}

Chitosan Nanoparticles, Mitomycin-C, Low Releasing Drug Kinetics, Intravesical Therapy, Bladder Tumor

\section{Introduction}

Bladder carcinoma is one of the most predominant malignancies of the urinary tract. Most bladder tumors are superficial at the diagnostics stage, since the symptoms are nonspecific and may be linked with many other conditions. Many urothelial cell carcinomas diagnoses are not invasive, since they go no deeper than the superficial layer (mucosa) of the bladder [1]. These tumors not only can be treated by surgical resection but also adjuvant intravesical installation of Bacille Calmette Guerine (BCG) and other chemotherapeutic agents including Mitomycin-C (MMC), Epirubicin, Doxorubicin and Adriamycin [2] [3] [4] [5]. Additionally, Mitomycin-C, Epirubicin and Doxorubicin can be used for a single, immediate, post-operative intravesical instillation after resection of the tumor [6] [7]. MMC is frequently used as an intravesical agent. However, the optimal dose usage and installation frequency of the MMC is somewhat controversial [8]. At present, some methods such as microwave-induced hyperthermia or electromotive drug administration, have been known to increase efficacy of MMC, however, the optimal usage of MMC has not been described [9]. It is further known that MMC should be administrated for an extended period with repeated dose administration to prevent of further tumor recurrences and progression. The optimal MMC molecule demonstrates certain characteristics, such as long-acting, reduced administration of repeated dose, reduced side-effect and low dose requirement, however there is no known ideal MMC molecule.

Drug delivery systems employ specific technologies that focus mainly on targeting release or delivery of drugs or any substance that is of medicinal importance. As an example, controlled drug release systems using anti-neoplastic drugs are currently being investigated by several researchers. The nanomaterial landscape is immense since well-characterized polymers, lipids, peptides and proteins, sugars, and surfactants that can be engineered into novel Nano formulation platforms. Liposomes, dendrimers, and nanogels have been used for both controlled drug delivery and cell growth scaffolds and are presented in many nanomedicines and will appear, to some extent, also in the nanomedicines in the future [10] [11]. Using advanced nanomaterials, such as functionalized quantum dots (QDs), nanoparticles can pave the way towards improved nano-bio systems with minimal resources, reduced health hazard and environmental friendly impacts [12] [13]. It has been further reported that major challenges in drug delivery, with synthesized drug delivery carriers' molecules, are their accumulations in the targeted cells, targeting a specific tissue with nuclear uptake and endosomal transfer [13]. Additionally, many other materials of therapeutic relevance, can cure 
cancer and other related diseases are under studies for targeted and time-release drug delivery. Some of these substances under current investigations include xanthan, gelatine, albumin and phospholipids [14].

Polymeric nanocarriers have paramount important in the field of science and have revealed many applications in the field of nanotechnology as well development in drug delivery systems. Nanocarriers have some specific properties which include size and shape, surface characteristics and chemical constituents that differs significantly with other polymers [15] [16]. Chitosan are highly basic polysaccharides that occur naturally with some specific properties which include the formation of polyoxysalt, formation of film in solution, optical structural properties and ability to chelate metal ions. Chitosan nanoparticles also have similar but more optimized properties than the normal chitosan solution, as revealed in a study that compared insulin chitosan solution and its nanoparticles, in which the nanoparticles showed more effective treatment in lowering the blood glucose level, more optical characteristics and its presence in the physiological environment is also less [17]. Chitosan nanoparticles was synthesized using a novel technique based on ionic gelation using sodium tripolyphosphate (TPP) as cross-linking agent TPP and the particles are known to have a different morphology and surface characteristic [18]. In this method, the TPP solution flows through the pores of a micro engineered membrane into the chitosan solution put in a stirred cell. The ionic cross-linking method is the most common among physical cross-linking techniques since the preparation procedure is simple, does not involve the use of organic solvent or high temperature, and no chemical interaction is involved. The advantages make this method efficient and safe for production of thermosensitive therapeutic agents such as proteins, peptides, hormones, and vaccines loaded into chitosan particulate systems. Assembly and formation of the particles is achieved by ionic cross- linking between chitosan and one of its derivatives, being cationic in nature, and either negatively charged macromolecules or anionic cross-linking agents. In some studies, the controlled drug delivery systems including MMC, Epirubicin and Doxorubicin has been performed by the synthesis with different nanogel and nanoparticles and the efficacy of drugs in the tissue can be increased [19] [20]. The muco-adhesive properties of these systems are an important factor in their retention and action in drug delivery. We aimed to synthesis a $\mathrm{pH}$ sensitive drug delivery system that will reduce the intravesical administration frequency with an enhanced efficacy of installation.

\section{Experimental}

\subsection{Materials}

Low viscous chitosan from shrimp shells [mol. wt. 50,000 - 190,000 Da (based on viscosity)] was purchased from Sigma Aldrich (USA). Glacial acetic acid (Glacial/27225/USA) solution was employed as a solvent for chitosan. Sodium tripolyphosphate (Alfa-Aesar 013440/USA) was used as a cross-linker. Phosphate Buf- 
fer Saline (PBS) was purchased from Fluka (Switzerland). Spectra/Por Dialysis membrane bag was used in vitro drug release studies (molecular weight cut-off was 12 - $14 \mathrm{kDa}$, Cole Parmer Ind.). MMC was obtained from Roche Diagnostics $\mathrm{GmbH}$ (Mannheim, Germany). All reagents were of analytical grade and used without further purification. Bladder cancer cell line T24 was sourced from the American Type Culture Collection (Manassas, VA). Dulbecco modified Eagle's medium (DMEM), 1\% penicillin/streptomycin, trypsin-EDTA and 10\% fetal bovine serum (FBS), were purchased from Thermo Fisher Scientific (MA, USA). MTT (4,5-Dimethylthiazol-2-yl)-2,5-Diphenyl tetrazolium bromide (MTT) kit was obtained from Sigma-Aldrich.

\subsection{The Synthesis of Chitosan Nanoparticles}

Chitosan nanoparticles were synthesized by using ionic gelation technique [18]. In a typical procedure, low viscous chitosan ( $\% 0.5 \mathrm{w} / \mathrm{v})$ was dissolved in $1 \%$ Glacial acetic acid solution. The $\mathrm{pH}$ of the prepared chitosan solution was adjusted to 4.5 by drop wise addition of $10 \mathrm{~N} \mathrm{NaOH}$. After $\mathrm{pH}$ adjusting, $4 \mathrm{~mL}$ of tripolyphosphate (TPP) $(0.25 \% \mathrm{w} / \mathrm{v})$ solution was dropped into $12 \mathrm{~mL}$ of chitosan solution. The resulting solution was mechanically stirred for $1 \mathrm{hr}$. at room temperature. The chitosan nanoparticles thus formed were recovered by centrifugation at $12.000 \mathrm{rpm}$ for $30 \mathrm{~min}$ and was subsequently washed three times followed by freeze drying for further studies.

\subsection{The Characterization of Chitosan Nanoparticles}

\section{Dynamic Light Scattering (DLS)}

After centrifugation, the supernatant was decanted and the nanoparticles were suspended in $2 \mathrm{ml}$ of distilled water using ultra sonication. The charge of the nanoparticle and their mean size were measured by using Dynamic Light Scattering (Malvern Instruments, Model 3000 HSA, England). The nanoparticle zeta potential was determined in distilled water. Analyses were performed in triplicate at room temperature.

Atomic Force Microscopy (AFM)

Morphological evaluations of chitosan nanoparticles were realized with an Atomic Force Microscope (AFM) (Nano magnetics, Turkey) that was used to obtain AFM images of nanoparticles. Samples were prepared by pouring the solutions on a mica surface. $20 \mu \mathrm{L}$ solution was dried at room temperature before analysis.

Fourier Transform Infrared Spectroscopy (FTIR)

Physicochemical characteristics of free drug MMC, chitosan nanoparticles and MMC encapsulated chitosan nanoparticles were analyzed by Shimadzu IR Prestige 21 model FT-IR spectroscopy.

\subsection{Loading Studies}

MCC is compatible with anionic agents. Different concentrations of MMC were 
dissolved in TPP solution $(0.25 \% \mathrm{w} / \mathrm{v})$ and then TPP solution was dropped into the chitosan solution. The recovery of the chitosan nanoparticles was done by centrifugation at $12,000 \mathrm{rpm}$ for $30 \mathrm{~min}$; then it was washed with distilled water several times and lyophilized.

\section{Drug Loading Efficiency (DLE)}

In other to determine the quantity of drug loaded, separation of nanoparticle from the aqueous suspension was done by centrifugation at $12.000 \mathrm{rpm}$ for 30 min. The amount of free MMC concentration in the supernatant solution was determined by measuring of absorbance values. (Thermo Scientific, Nanodrop 1000 ) at a wavelength of $358 \mathrm{~nm}$. DLE were calculated according to the following equation.

$D L E(\%)=\frac{[\text { Concentration of initial Mitomycin C }- \text { Concentration of free Mitomycin C in supernatant }]}{\text { Concentration of initial Mitomycin C }} \times 100$

MMC was also confirmed by the FTIR studies.

\subsection{In Vitro Release Studies}

In vitro release analysis was carried out in a double-cell system mimicking the urination system including a dialysis membrane bag (molecular weight cut-off was $12-14 \mathrm{kDa}$ ) between the cells. $5 \mathrm{ml}$ of phosphate buffer solution $(\mathrm{pH}=7.4$ ) was put into one side and drug loaded lyophilized nanoparticles as well as specific quantity of MMC were suspended in $5 \mathrm{ml}$ PBS and put into another cell. During the drug loading and release studies, the initial amount of MMC was changed (i.e., $0.5,1.25$ and $2.5 \mathrm{mg}$ ) to get different release profiles. The nanoparticle samples were placed in a water bath with mild agitation and were set a temperature of $37^{\circ} \mathrm{C}$. At particular time intervals of 30, 60, 90, 120, 150, 180, 240, 300, 360 min etc.; $100 \mu \mathrm{L}$ released medium was taken out from the nanoparticlefree cell and the same volume fresh PBS solution was added. The content of released MMC was detected using Ultraviolet Spectrometer (Thermo Scientific, Nanodrop 1000) with a wavelength of $358 \mathrm{~nm}(n=6)$. Cumulative amount of drug (in \%) released from the nanoparticles was calculated. The amount of MMC in the solution was calculated by creating a calibration curve with known amounts of drug concentrations. All of the release studies were repeated $(n=6)$. Additionally, MMC release studies have examined for different $\mathrm{pH}$ values. The $\mathrm{pH}$ of PBS solution was adjusted to 6 and $7.4(n=6)$. During the $\mathrm{pH}$ experiments, the nanoparticles were loaded with $1.25 \mathrm{mg}$ MMC.

\subsection{In Vitro Cytotoxicity Studies}

The standard MTT assay was performed to determine bare NPs and drug loaded nanoparticle toxicity. T24 cells were cultured in DMEM medium with additional $1 \%$ penicillin/streptomycin mixture and 10\% FBS. Briefly, T24 cells were seeded onto a 96-well plate at a density of $1 \times 10^{4}$ cells/well per well. After $24 \mathrm{~h}$, the medium was removed and the cells were treated with a range of concentrations at $0.5,1.25$ and $2.50 \mathrm{mg} / \mathrm{ml}$ of MMC, bare NPs and MMC/CS nanoparticles. The 
untreated cells were accepted as a control. After $24 \mathrm{~h}$ incubation, $200 \mu \mathrm{L}$ of 3 $\mathrm{mg} / \mathrm{ml} \mathrm{MTT} \mathrm{solution} \mathrm{was} \mathrm{added} \mathrm{to} \mathrm{each} \mathrm{well} \mathrm{and} \mathrm{the} \mathrm{plate} \mathrm{was} \mathrm{incubated} \mathrm{at}$ $37^{\circ} \mathrm{C}, 5 \% \mathrm{CO}_{2}$ for $4 \mathrm{~h}$. DMEM was refreshed at $2,4,6,8,10,12$ and $24 \mathrm{~h}$ to mimic the urination discipline. The absorbance was measured using an absorbance micro plate reader (Thermo Fisher Scientific, 1510, Finland) at a wavelength of $450 \mathrm{~nm}$. The viability of the cell was deducted by adopting the following equation:

$$
\text { Viability percentage }=\frac{A T}{A U} \times 100
$$

where $A T$ is the Absorbance of treated cells and $A U$ is Absorbance of untreated cells. The cell viability graph is drawn using the mean absorbance of three independent replicates.

\subsection{Statistical Analysis}

All measurements were calculated as the mean \pm standard deviation (SD). Oneway analysis of variance (ANOVA) was adopted in the analysis of the results with the Student-Newman-Keuls multiple comparisons or t-test when comparing the differences between the means of two groups at the same time point. The statistical significance was benched at $<0.05$ probability.

\section{Results and Discussion}

\subsection{Size Distribution of CS and MMC/CS Nanoparticles}

In this study, chitosan nanoparticles syntheses were carried out through ionic gelation technique and MMC was loaded successfully to these nanoparticles. Morphological evaluation showed that the obtained nanoparticles were sphere like in shape with a narrow size distribution range at around $140 \mathrm{~nm}$ as shown in dynamic light scattering output and the polydispersity $(\mathrm{Q})$ was 0.370 . It is further shown that the obtained nanoparticles were sphere like in shape with a narrow size distribution range at around $140 \mathrm{~nm}$ as shown in dynamic light scattering output. The size of chitosan nanoparticles was smaller than $200 \mathrm{~nm}$, which due to the chitosan nanoparticle size, allows for targeting and accumulation in cancerous tissues [21]. Such a range of nanoparticles may accumulate more accurately in tumor due to the enhanced permeability and retention (EPR) effect, which is often regarded as "passive" targeting [22].

As shown in Table 1, polydispersity indices of chitosan nanoparticles (CS) and MMC encapsulated chitosan nanoparticles (MMC/CS) were lower than 0.4, indicating the size distributions of nanoparticles were narrow and formulations of nanoparticle were mono dispersed in size distribution. Compared to the size results of nanoparticles and drug carrying nanoparticles, the encapsulation of MMC moderately increased the size of the nanoparticles as the ionic interaction that exist among the chitosan TPP can be affected by drug loading. Drug anions can engage the CS cations due to the hydrophilicity of MMC in aqueous solution. This on the other hand can hamper the CS and TPP interaction. This may 
Table 1. Z-average zeta-potential and particle size of the drug encapsulated CS nanoparticles.

\begin{tabular}{cccc}
\hline Sample & Size $(\mathrm{nm})$ & Polydispersity & Zeta-potential $(\mathrm{mV})$ \\
\hline CS & $140 \pm 5$ & $0.370 \pm 0.055$ & +32.6 \\
MMC/CS nanoparticles $^{\mathrm{a}}$ & $176 \pm 9$ & $0.282 \pm 0.046$ & +26.8 \\
MMC/CS nanoparticles $^{\mathrm{b}}$ & $199 \pm 11$ & $0.333 \pm 0.074$ & +25.1 \\
MMC/CS nanoparticles $^{\mathrm{c}}$ & $210 \pm 14$ & $0.326 \pm 0.055$ & +23.8 \\
\hline
\end{tabular}

${ }^{\mathrm{a}} 0.5 \mathrm{mg} / \mathrm{ml},{ }^{\mathrm{b}} 1.25 \mathrm{mg} / \mathrm{ml},{ }^{\mathrm{c}} 2.5 \mathrm{mg} / \mathrm{ml}$.

be more advantageous and usable. We think that these nanoparticles have some advantage such as easy and simple administration, easier and simpler contact to bladder epithelium. Therefore, this MMC molecules synthesis with chitosan nanoparticles should be more suitable for a low drug releasing system. However, further studies will further examine the advantage of nanoparticles.

Particle size and zeta potential are two major parameters for assessing nanoparticle stability. As particle size ensures the spread of nanoparticles around the body, its stability is governed by zeta potential. Nanoparticles with zeta potential above $\pm 30 \mathrm{mV}$ show relatively stable nanosuspension [23]. Also, it’s been proven that the nanoparticles intra-cellular distribution is affected by its zeta potential affect [24]. As the Zeta potential of the nanoparticle increases, their stability also increases. Furthermore, it's been proven that the nanoparticles charge density contributes to its binding to cancer cells. Therefore, charged nanoparticles are ideal for cancer treatment. To show the stability of nanoparticles, the zeta potential was investigated. In our study, the zeta potential values of chitosan nanoparticles were identified to be $+32 \mathrm{mV}$, indicating net positive surface charge. Zeta potential decreased with the increasing of MMC amount, while the diameter of the drug encapsulated nanoparticles increased with loading of MMC. This showed that the increase in the amount of MMC reduced the interaction among TPP and $\mathrm{CS}$, resulting in less compact structures.

\subsection{Morphology}

Figure 1 shows morphological characteristics and cross-section diameter distribution of Chitosan nanoparticles through AFM images. The AFM images indicated the smooth regular and spherical surfaces of all nanoparticle formulations. As assessed by AFM, the nanoparticles were well separated and no aggregation was observed. The mean diameters of nanoparticles obtained by AFM were less than the values measured by DLS due to the dried, dehydrated and shrunk nature of nanoparticles.

\subsection{Surface Chemistry of CS and MMC/CS Nanoparticles}

The FTIR results revealed the structural properties of MMC and MMC loaded chitosan nanoparticles (Figure 2). It further demonstrates the comparison between unloaded and loaded nanoparticles. The minor changes in the absorption 


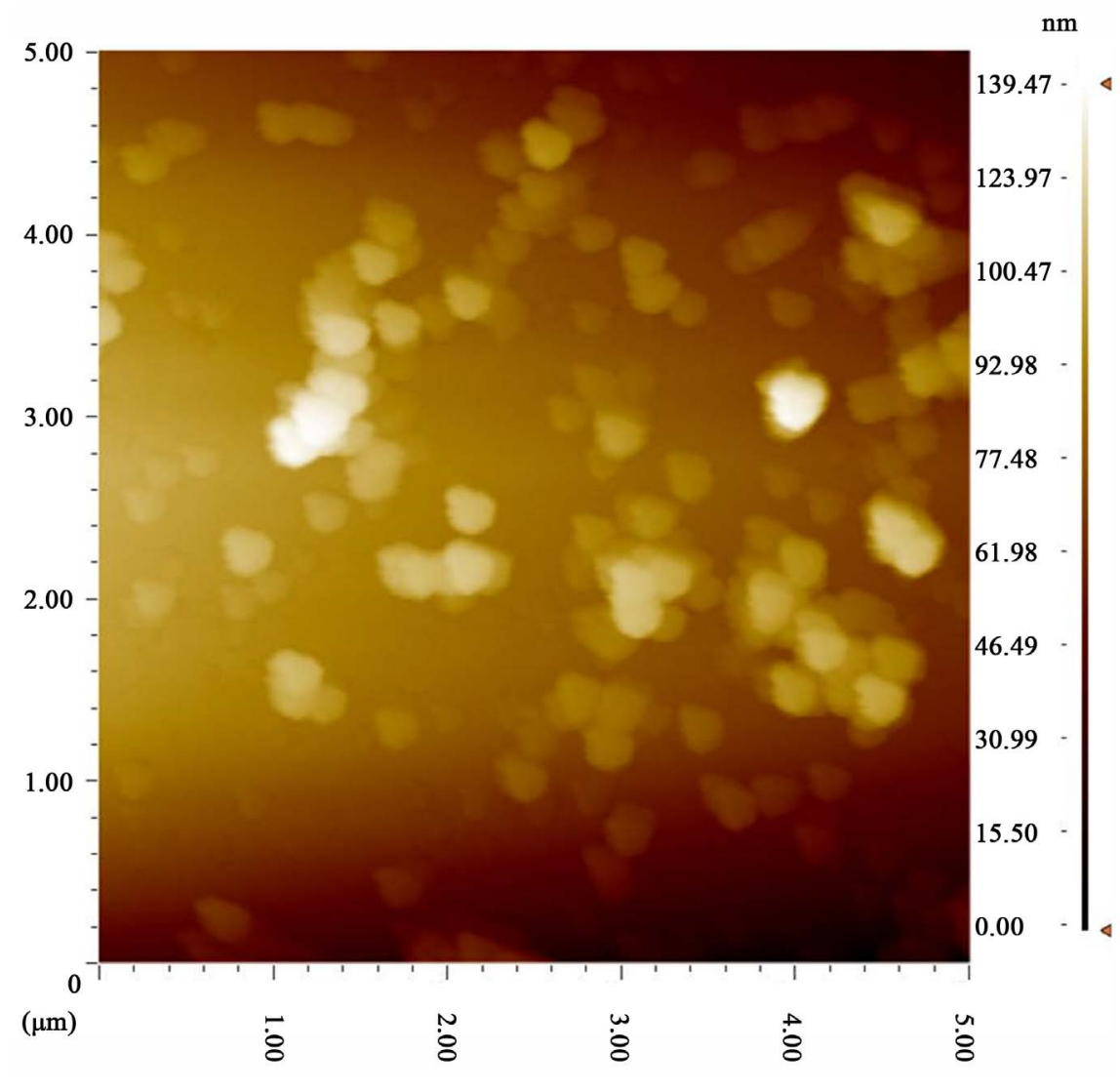

(a)

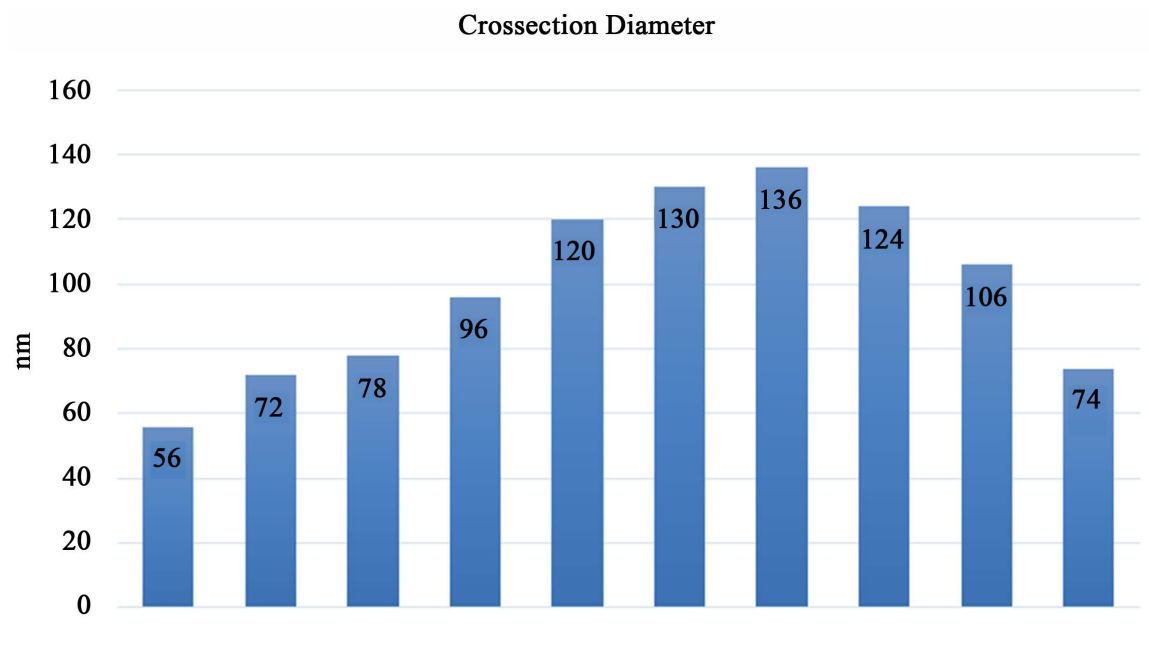

$0.02-0.1 \quad 0.1-0.14 \quad 0.14-0.16 \quad 0.16-0.2 \quad 0.2-0.28 \quad 0.28-0.340 .34-0.440 .44-0.520 .52-0.580 .58-0.66$

$\mu \mathrm{m}$

(b)

Figure 1. (a) AFM image of chitosan nanoparticles. Chitosan nanoparticles were synthesized by using ionic gelation technique. AFM images were obtained by Nano magnetics Equipment, Turkey, using mica surface. The images were adjusted in the $\mathrm{X}$ and $\mathrm{Y}$ plane, Scanning area of $5 \mu \mathrm{m} \times 5 \mu \mathrm{m}$; (b) Diameter distribution of chitosan nanoparticles. The AFM image of chitosan nanoparticles in the solutions showed a mean average size under $140 \mathrm{~nm}$ that was adapted with the result of DLS showed in Table 1. 


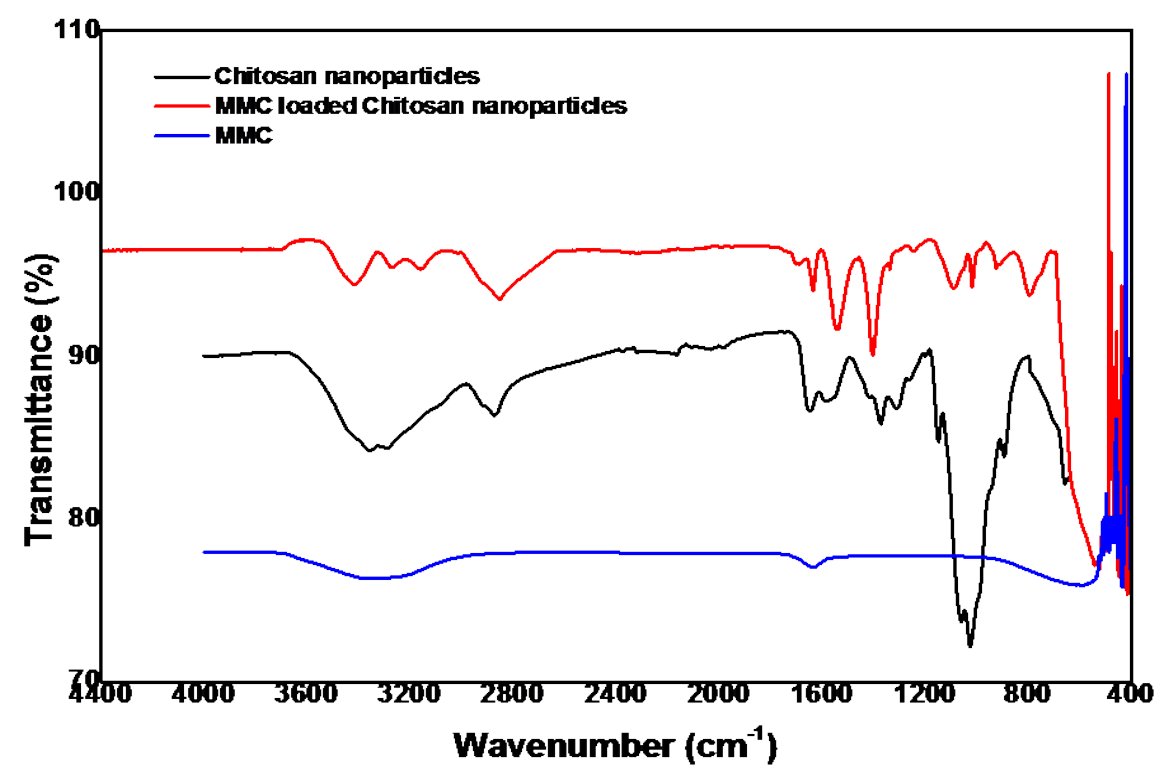

Figure 2. FTIR spectra of chitosan nanoparticles, MMC loaded chitosan nanoparticles and free MMC drug.

peaks usually represent the bonds in the chitosan that are responsible for stretching in the sugar ring and the bonds included hydrogen bonds, Van der Waals forces, and dipole moments. The change in the peaks indicates the involvement of $\mathrm{O}-\mathrm{H}$ or $\mathrm{N}-\mathrm{H}$ bond in the synthesis as reported by Ramanet et al. [25]. The peak at $1600 \mathrm{~cm}^{-1}$ indicated the stretching vibration between the sugar ring and the $\mathrm{N}-\mathrm{H}$ or $\mathrm{O}-\mathrm{H}$ group in the synthesis of MMC and the chitosan nanoparticles. Furthermore, the peak change at about $1625 \mathrm{~cm}^{-1}$ clearly reveals the functional group found in the MMC and MMC loaded chitosan nanoparticles and it was absent in chitosan nanoparticles without MMC, representing the contribution of the functional groups of MMC. The FTIR results agree with work done by Dongwei et al. [26]. There was a change between 3600 and $2800 \mathrm{~cm}^{-1}$ observed peaks due to the ingestion of the MMC. Besides, the observation of only the $1020 \mathrm{~cm}^{-1}$ peak of chitosan nanoparticles in drug loaded nanoparticles is an indication of the same week physical interactions between the drug and the particles. The FTIR spectra reveals that bonds present in the sugar ring of chitosan may be accountable for the synthesis of MMC loaded chitosan nanoparticles.

\subsection{Determination of Drug Loading Efficiency}

The intravesical therapy aims to eliminate the active tumor cells which have the probability of implantation in the bladder mucosa after tumor resection. MMC usually preferred for the intravesical chemotherapy, is an alkylating agent with the molecular weight of $334 \mathrm{kDa}$. It is an antineoplastic, and inhibits the synthesis of DNA [27]. Although it is not clear, it is usually suggested the dose of MMC is $40 \mathrm{mg}$ per week intravesical (total 6 - 8 weeks). This administration of MMC has several disadvantages such as adverse effect due to repeated installation, need for administration weekly and frequent hospital visits. An ideal approach is 
to decrease the dose and frequency of MMC administration. Alternatively, the frequency of administration and drug dose should be optimized for the treatment of superficial bladder tumors. At the present time, there is no ideal form of MMC for intravesical chemotherapy. In this study, a low in vitro drug release system was performed with the synthesized MMC encapsulated chitosan nanoparticle. The amount of released MMC molecule was evaluated using a PBS system for the different doses of loaded MMC at neutral $\mathrm{pH}$. Results were compared with the standard (nonencapsulated) MMC molecules. MMC is a well-known chemotherapeutic agent because of its antitumor antibiotic potential and was chosen as the antitumor drug model in this work. MMC is a highly hydrophilic drug hence, it possesses high affinity to an aqueous phase and drug release is very rapid.

In our study, Table 2 shows the entrapment efficiency (EE) and drug-loading efficiency (LE) of MMC with three different initial concentrations $0.5 \mathrm{mg} / \mathrm{ml}$, $1.25 \mathrm{mg} / \mathrm{ml}$ and $2.5 \mathrm{mg} / \mathrm{ml}$ into chitosan nanoparticles were $55 \%, 47 \%, 36 \%$ and 19\%, 25\%, 39\% respectively (Table 2). This disclosed well continued release execution, efficient, and loading capacity [28]. It was seen that the high drug loading resulted in low drug encapsulation efficiency [29]. When the drug concentration was increased, the nanoparticulate system showed tendency to protrude the drug outside.

\subsection{In Vitro Drug Release}

The release profiles of MMC-CS nanoparticles at varying $\mathrm{pH}$ values showed that for all the nanoparticle formulations, there was an initial fast release of the drugs, but it tends to slow down. Over longer period CS nanoparticles released $47 \%$ and $53 \%$ at $\mathrm{pH}$ of 6.0 and 7.4 respectively ( $p: 0.012$ ). Therefore, $\mathrm{pH}$ of 7.4 conditions lead to the maintaining of MMC stability. Previous studies have reported that MCC are liable to denaturation when placed in acidic medium [30]. This poses as one of the setbacks to the use of MMC for intravesical drug delivery with MMC due to its denaturation to acid which leads to possible loss of the therapeutic dosage. This, on the other hand may hamper the unloading of the drug in the release medium (Phosphate buffer, $\mathrm{pH}$ 6.0), thereby leading to lower MMC release percentage due to the degradation of the release free drug. In this study, formed in vitro system, we concluded that the higher $\mathrm{pH}$, the higher are the released MMC molecules with synthesized chitosan nanoparticles. Because

Table 2. Entrapment efficiency (EE) and drug-loading efficiency (LE) with different drug concentration.

\begin{tabular}{ccc}
\hline Sample & Entrapment efficiency (EE) & Drug-loading efficiency (LE) \\
\hline MMC/CS nanoparticles $^{\mathrm{a}}$ & $54 \pm 0.45$ & $18 \pm 0.83$ \\
MMC/CS nanoparticles $^{\mathrm{b}}$ & $47 \pm 0.11$ & $24 \pm 0.78$ \\
MMC/CS nanoparticles $^{\mathrm{c}}$ & $36 \pm 14$ & $38 \pm 0.61$ \\
\hline
\end{tabular}

${ }^{\mathrm{a}} 0.5 \mathrm{mg} / \mathrm{ml},{ }^{\mathrm{b}} 1.25 \mathrm{mg} / \mathrm{ml},{ }^{\mathrm{c}} 2.5 \mathrm{mg} / \mathrm{ml}$. 
of high number of molecules, the efficiency of drug may be higher. So that, this result shows urinary alkalization may contribute to the efficiency of MMC molecules synthesized chitosan nanoparticles. Is known that the efficiency of conventionally intravesical MMC can be increased by orally alkalinizing agent such as potassium citrate [31]. In our in vitro system, when the urinary $\mathrm{pH}$ is increased by orally alkalinizing agent, the efficiency of MMC molecules with synthesized chitosan nanoparticles may be more than conventionally intravesical MMC. It can be concluded that according to our hypothesis, this drug system can be adjusted to low releasing and can be modified of $\mathrm{pH}$ by orally alkalinizing agents. Therefore, this system should provide a more effective treatment modality than conventional intravesical MMC for superficial bladder cancer. Theoretically, most drugs often remain in the carrier for a specific set time with regards to appropriate physiological conditions of $\mathrm{pH}$ 7.4, proving the ability of retaining drugs in the blood circulation for a longer time period, while vastly reducing the possible side effect to healthy tissues. Also, once the chitosan nanoparticles that have been loaded with drugs are absorbed by tumor cells via endocytosis processes, there is a possibility of faster release at lower locale $\mathrm{pH}$, for instance, surrounding the tumor site or lysosome and inside of the tumor cells, resulting in vital improvement in the treatment of cancer [32] (Figure 3).

In the release studies, it was obvious that drug release was significantly affected by the earlier concentration of drug [31]. MMC/CS had a longer time release than free MMC. At the end of the 1440 min. period, 44\%, 53\% and 65\% MMC was released from loading doses in $7.4 \mathrm{pH}$ PBS solution for $0.5 \mathrm{mg} / \mathrm{mL}$, $1.25 \mathrm{mg} / \mathrm{mL}$ and $2.5 \mathrm{mg} / \mathrm{mL}$ respectively ( $p: 0.018$ ). Whereas $99.08 \%$ of free MMC was released towards the end of the 120 minutes; and the drug was completely released in 150 minutes. At the end of the 1440 minute, free MMC was not present in the medium.

Explanation to this is related to the nanoparticle size and its zeta potential as a larger nanoparticle is a function of increase in MMC concentration and decrease

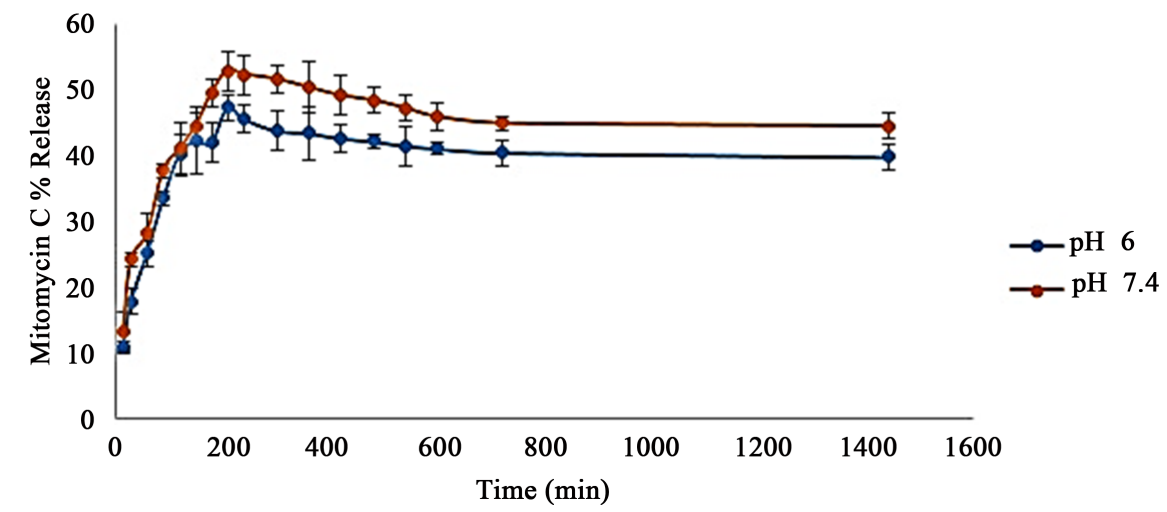

Figure 3. Release profiles of MMC from chitosan nanoparticles in different $\mathrm{pH}$ values. Release studies were carried out by using two different release medium, phosphate buffers at $\mathrm{pH} 7.4$ and $\mathrm{pH} 6$ in order to simulate the physiological condition and urinary condition respectively. 
of its Zeta potential, and depicts an intense interaction that exist between the polymer matrix and drugs. Nanoparticles that are loaded with drugs of varying initial low concentration tend to release drugs much faster because of surface area to volumetric ratio. Furthermore, when compared to nanoparticles much smaller in size, diffusion of the drug out of the inside of the nanoparticle with a large diameter size takes longer time interval; hence leading to much slower release of MMC. Gam and Wang [33], reported a similar drug release pattern with BSA loaded chitosan nanoparticles (Figure 4).

The nanoparticle release of drug relied on the swelling process rather than erosion or degradation. It is predicted that $100 \%$ drug release can be realized in the colon after $4-5 \mathrm{hrs}$ with the presence of enzyme. Also, the method of release demonstrates that collective drug release can be adjusted by varying the initial concentration along with the $\mathrm{pH}$ of the release medium.

\subsection{In Vitro Cytotoxicity Study}

Mitomycin is a cancer drug that is currently used intravesically for the rapid treatment of bladder cancer. Thus, it is extremely important to ascertain the effect of mitomycin nanoparticles formulation against different cancer cell models. Cytotoxic effect of free drugs, CS nanoparticls and three different dosages of MMC loaded CS nanoparticles against human bladder cell lines T24 were ascertained by MTT assay as shown in Figure 5 .

Different nanoparticles formulations, concentrations and dosages (12.5, 25 and $50 \mu \mathrm{g} / \mathrm{l})$ were used against T24 cells after $24 \mathrm{hr}$ treatment. From the result presented in Figure 5, different dosages $(12.5,25$ and $50 \mu \mathrm{g} / \mathrm{l})$ of free MMC showed clear reduction in cell viability in comparison to the control (Untreated cells). Also, the cytotoxicty of free MMC started at lower dosage $12.5 \mu \mathrm{g} / \mathrm{l}$ but

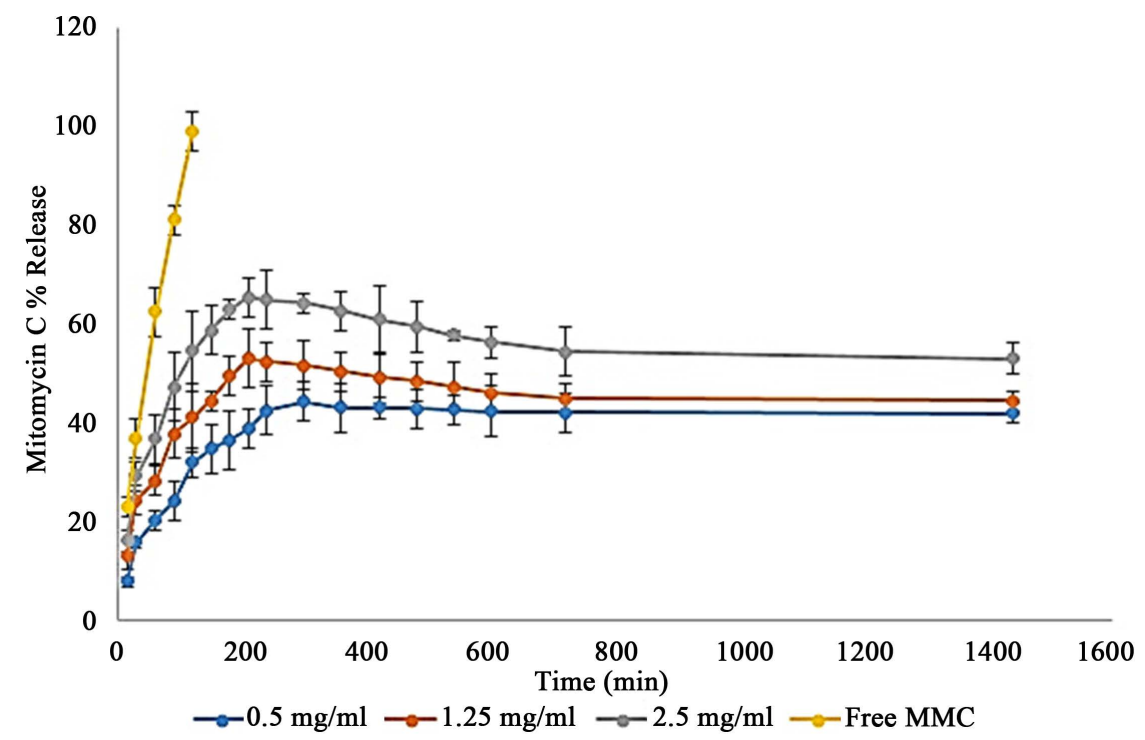

Figure 4. Release profiles of MMC from chitosan nanoparticles in different initial loading doses and the cumulative release of free MMC. Data are represented as mean $\pm S E M, n=$ 6. 


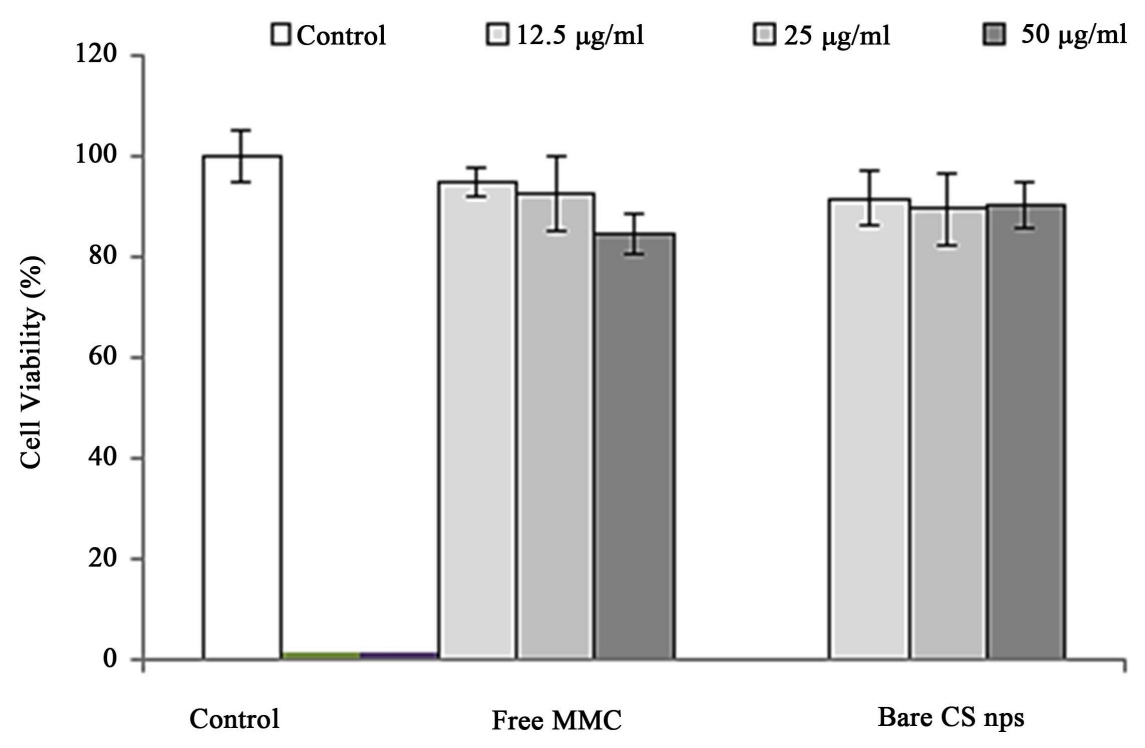

(a)

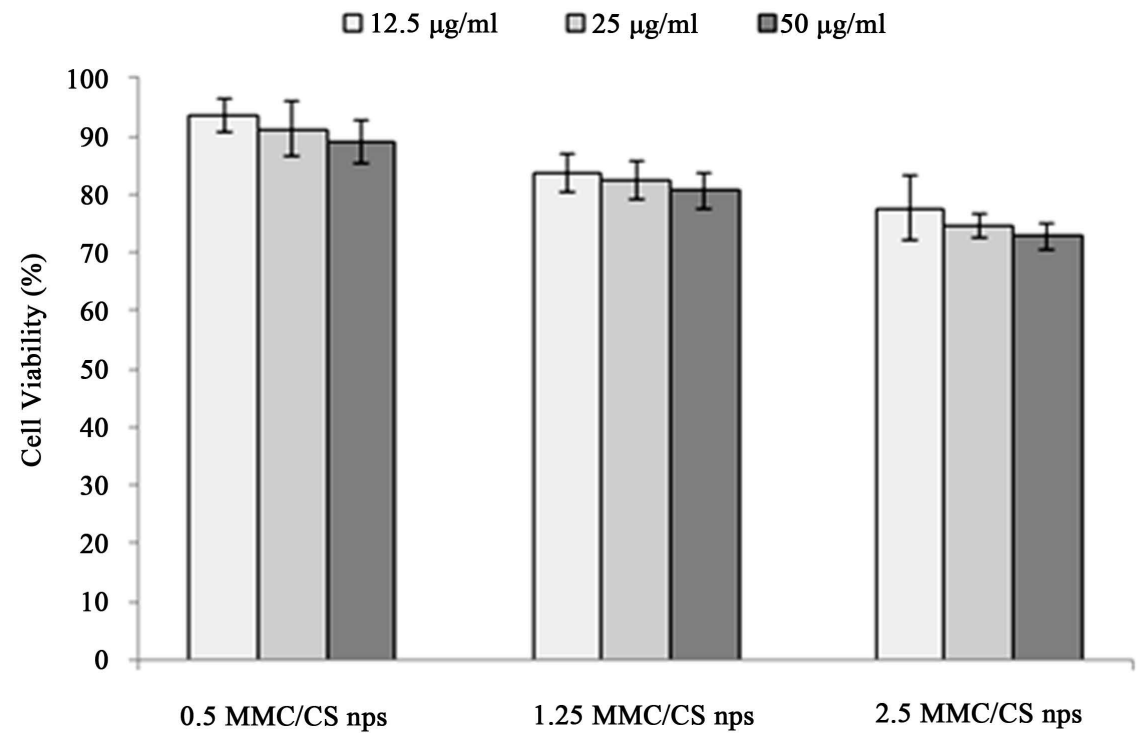

(b)

Figure 5. Effect of free MMC, CS nanoparticles (a) and different dosage of MMC loaded CS nanoparticles on viability of human bladder cancer cell lines T24 after 24 hr. treatment using MTT Assay. Experiment data is presented as mean \pm SEM of at least replicates analysis $(n \geq 3)$.

showed more decrease at $50 \mu \mathrm{g} / \mathrm{l}$ and it revealed that, the cytotoxicity of free MMC is completely dose dependent. It has been reported that, the activity of Mitomycin C against different human cancer lines has to do with inactivation of gluthathione S-transferase and also activation of DT-diaphorase [34]. Also, one of the most active components in Mitomycin C that has anti-cancer activity has shown about $20 \%$ response rate and study was carried out against gastro-intestinal cancers [35]. Mitomycin can also induced cytotoxicity in cancer cells by decreasing Rad51 expression and activation of AKT [36]. 
The native CS nanoparticles also showed decrease in cell viability upon comparison with the untreated cells but in this case all the dosages $(12.5,25$ and 50 $\mu \mathrm{g} / \mathrm{l})$ almost revealed similar effect. The cytotoxic effect in Bare CS nps is not dose dependent as in free MMC which showed more decreased in cell viability as the dose increased. The results of this study are in agreement with the work done by Deng et al. [37] which revealed that nanoparticles are very prominent and effective in the treatment of cancer independent of the dosage.

The activity of different concentration of MMC/CS nps $(0.5,1.25$ and $2.5 \mu \mathrm{g} / \mathrm{l})$ were also studied against the same cell lines and three different dosages were used. And the result showed promising decrease in cell viability with an increase in concentration. The highest concentration used showed more cytotoxic effect than the previous lower doses. Chitosan is very useful in the treatment of many diseases, more especially in cancer therapy. And as a native polysaccharides when combined with highly anti-cancer drugs, it will serve as a carrier for that drug to achieve an efficient treatment [38]. A combination of Mitomycin C and Chitosan nanoparticles revealed a decrease in the cell viability and is dose dependent.

\section{Conclusion}

In the treatment of superficial bladder cancer, MMC can be used instead of BCG (Bacille-Calmette Guerine) which has very high side effect. Synthesized nanoparticles, notwithstanding excellent association with MMC, are infrequently used for long term efficacy. In this investigation, ionic gelation technique was adopted to produce anticancer polymeric nanoparticles containing MMC using the high solubility of MMC in water, with great impact on the zeta potential, efficiency of encapsulation and the particle size of CS nanoparticles. Studies of the in vitro drug release reveal a steady state release of MMC by the nanoparticles. Nanoparticles that are bioadhesive are intravesically introduced into the body with ease, as the coated bioadhesives make it possible for adhesion of nanoparticles to the walls of the bladder. Since CS possess hydrophilic surfaces and are biocompatible, they can stay for a longer time period in the blood stream. In line with the findings of this study, it is concluded that the drug encapsulated chitosan nanoparticles that were synthesized possess higher ability to be adopted as a $\mathrm{pH}$ sensitive drug delivery system and hence show a great promise for intravesical installation.

\section{References}

[1] Cui, J., Wang, W., Chen, S., Chen, P., Yang, Y., Guo, Y., Zhu, Y., Chen, F. and Shi, B. (2016) Combination of Intravesical Chemotherapy and Bacillus Calmette-Guerin versus Bacillus Calmette-Guerin Monotherapy in Intermediate- and High-Risk Nonmuscle Invasive Bladder Cancer: A Systematic Review and Meta-analysis. Medicine, 95, Article No: e2572. https://doi.org/10.1097/MD.0000000000002572

[2] Zargar, H., Aning, J., Ischia, J., So, A. and Black, P. (2014) Optimizing Intravesical Mitomycin C Therapy in Non-Muscle-Invasive Bladder Cancer. Nature Reviews 
Urology, 11, 220-320. https://doi.org/10.1038/nrurol.2014.52

[3] Lightfoot, A.J., Breyer, B.N., Rosevear, H.M., Erickson, B.A., Konety, B.R. and O’Donnell, M.A. (2014) Multi-Institutional Analysis of Sequential Intravesical Gemcitabine and Mitomycin C Chemotherapy for Non-Muscle Invasive Bladder Cancer. Urologic Oncology, 32, 35.e.15-35.e.9.

[4] Porten, S.P., Leapman, M.S. and Greene, K.L. (2015) Intravesical Chemotherapy in Non-Muscle-Invasive Bladder Cancer. Indian Journal of Urology, 31, 297-303. https://doi.org/10.4103/0970-1591.166446

[5] Sylvester, R.J., Oosterlinck, W. and Van der Meijden, A. (2004) A Single Immediate Postoperative Instillation of Chemotherapy Decreases the Risk of Recurrence in Patients with Stage Ta T1 Bladder Cancer: A Meta-Analysis of Published Results of Randomized Clinical Trials. The Journal of Urology, 171, 2186-2190. https://doi.org/10.1097/01.ju.0000125486.92260.b2

[6] Abern, M.R., Owusu, R.A. and Anderson, M.R. (2013) Perioperative Intravesical Chemotherapy in Non-Muscle-Invasive Bladder Cancer: A Systematic Review and Meta-Analysis. Journal of the National Comprehensive Cancer Network, 11, 477-484. https://doi.org/10.6004/jnccn.2013.0060

[7] Sylvester, R.J., Oosterlinck, W. and Witjes, J.A. (2008) The Schedule and Duration of Intravesical Chemotherapy in Patients with Non-Muscle-Invasive Bladder Cancer: A Systematic Review of the Published Results of Randomized Clinical Trials. European Urology, 53, 709-719. https://doi.org/10.1016/j.eururo.2008.01.015

[8] Lammers, R.J., Witjes, J.A., Inman, B.A., Leibovitch, I., Laufer, M., Nativ, O. and Colombo, R. (2011) The Role of a Combined Regimen with Intravesical Chemotherapy and Hyperthermia in the Management of Non-Muscle-Invasive Bladder Cancer: A Systematic Review. European Urology, 60, 81-93.

https://doi.org/10.1016/j.eururo.2011.04.023

[9] Di Stasi, S.M., Giannantoni, A., Giurioli, A., Valenti, M., Zampa, G., Storti, L., Attisani, F., De Carolis, A., Capelli, G. and Vespasiani, G. (2006) Sequential BCG and Electromotive Mitomycin versus BCG Alone for High-Risk Superficial Bladder Cancer: A Randomized Controlled Trial. The Lancet Oncology, 7, 43-51. https://doi.org/10.1016/S1470-2045(05)70472-1

[10] Duncan, R. (2003) The Dawning Era of Polymer Therapeutics. Nature Reviews Drug Discovery, 2, 347-360. https://doi.org/10.1038/nrd1088

[11] Ferrari, M. (2005) Cancer Nanotechnology: Opportunities and Challenges. Nature Reviews Cancer, 5, 161-171. https://doi.org/10.1038/nrc1566

[12] Pieter, V. and Raymond, M.S. (2016) Biologically-Inspired Drug Delivery Systems. Advanced Drug Delivery Reviews A, 106, 1-2.

[13] Vaseashta, A. (2008) Functionalized Nanoscale Materials, Devices and Systems, NATO Science for Peace and Security Series B: Physics and Biophysics. Springer, New York.

[14] Akbal, Ö., Erdal, E., Vural, T., Kavaz, D. and Denkbas, E.B. (2017) Comparison of Protein- and Polysaccharide-Based Nanoparticles for Cancer Therapy: Synthesis, Characterization, Drug Release, and Interaction with a Breast Cancer Cell Line, Artificial Cells. Nanomedicine, and Biotechnology, 45, 193-203. https://doi.org/10.3109/21691401.2016.1170694

[15] Kavaz, D., Odabas, S., Denkbas, E.B. and Vaseashta, A. (2012) A Practical Methodology of IgG Purification via Chitosan Based Magnetic Nanoparticles. Digest Journal of Nanomaterials and Biostructures, 7, 1165-1177. 
[16] Rampino, A., Borgogna, M., Blasi, P., Bellich, B. and Cesaro, A. (2013) Chitosan Nanoparticles: Preparation, Size Evolution and Stability. International Journal of Pharmaceutics, 455, 219-228.

[17] Dyer, M., Hinchcliffe, M., Watts, P., Castile, J., Jabbal-Gill, I., Nankervis, R., Smith, A. and Illum, L. (2002) Nasal Delivery of Insulin Using Novel Chitosan Based Formulations: A Comparative Study in Two Animal Models between Simple Chitosan Formulations and Chitosan Nanoparticles. Pharmaceutical Research, 19, 998-1008. https://doi.org/10.1023/A:1016418523014

[18] Kavaz, D., Odabas, S., Guven, E., eMobile, M. and Denkbas, E.B. (2010) Bleomycin Loaded Magnetic Nanoparticles as Multifunctional Nanocarriers. Journal of Bioactive and Compatible Polymers, 25, 305-318. https://doi.org/10.1177/0883911509360735

[19] Rafiee, E. and Eavani, S. (2014) PH-Responsive Controlled Release of Epirubicin from Fe@Si-Pw Hybrid Nanoparticles. Materials Science and Engineering C: Materials for Biological Applications, 39, 340-343.

[20] Yu, Y., Chen, C.K., Law, W.C., Weinheimer, E., Sengupta, S. and Prasad, P.N. (2014) Polylactide-Graft-Doxorubicin Nanoparticles with Precisely Controlled Drug Loading for pH-Triggered Drug Delivery, S. Biomacromolecules, 15, 524-532. https://doi.org/10.1021/bm401471p

[21] Zhang, Y., Li, J., Lang, M., Tang, X., Li, L. and Shen, X. (2011) Folate-Functionalized Nanoparticles for Controlled 5-Fluorouracil Delivery. Journal of Colloid and Interface Science, 354, 202-209.

[22] Vivek, R., NipunBabu, V., Thangam, R., Subramanian, K.S. and Kannan, S. (2013) $\mathrm{PH}-$ Responsive Drug Delivery of Chitosan Nanoparticles as Tamoxifen Carriers for Effective Anti-Tumor Activity in Breast Cancer Cells. Colloids and Surfaces B: Biointerfaces, 111, 117-123.

[23] Vishvajit, A.K., Deepali, M.J. and Vilasrao, J.K. (2010) Nanosuspension a Novel Drug Delivery System. International Journal of Pharma and Bio Sciences, 1, 352-360.

[24] Panyam, J., Zhou, W.Z., Prabha, S.K. and Labhasetwar, V. (2002) Rapid Endo-Lysosomal Escape of Poly(DL-lactide-co-glycolide) Nanoparticles: Implications for Drug and Gene Delivery. The FASEB Journal, 16, 1217-1226.

https://doi.org/10.1096/fj.02-0088com

[25] Raman, S., Kandula, M.P., Jacob, J.A., Soundararajan, K., Ramar, T., Palani, G., Muthukalingan, K. and Shanmugam, A. (2012) Cytotoxic Effect of Green Synthesized Silver Nanoparticles Using Melia Azedarach against in Vitro HeLa Cell Lines and Lymphoma Mice Model. Process Biochemistry, 47, 273-279.

[26] Dongwei, W. and Weiping, Q. (2008) Facile Synthesis of Ag and Au Nanoparticles Utilizing Chitosan as a Mediator Agent. Colloids and Surfaces, 62, 136-142.

[27] McEvoy, G.K. (2007) AHFS 2007 Drug Information Essentials. American Society of Health-System Pharmacists, Inc., Bethesda, 1139-1141.

[28] Shi, Y., Wan, A., Shi, Y., Zhang, Y. and Chen, Y. (2014) Experimental and Mathematical Studies on the Drug Release Properties of Aspirin Loaded Chitosan Nanoparticles. BioMed Research, 2014, Article ID: 613619.

[29] Liu, Y., Li, K., Liu, B. and Feng, S.S. (2010) A Strategy for Precision Engineering of Nanoparticles of Biodegradable Copolymers for Quantitative Control of Targeted Drug Delivery. Biomaterials, 31, 9145-9155.

[30] Beijnen, J.H., Wiese, G. and Underberg, W.J. (1985) Aspects of the Chemical Stabil- 
ity of Doxorubicin and Seven Other Anthracyclines in Acidic Solution. Pharmaceutisch Weekblad, Scientific Edition, 21, 109-116. https://doi.org/10.1007/BF01968712

[31] Au, J.L., Badalament, R.A., Wientjes, M.G., Young, D.C., Warner, J.A., Venema, P.L., Pollifrone, D.L., Harbrecht, J.D., Chin, J.L. and Lerner, S.P. (2001) Methods to Improve Efficacy of Intravesical Mitomycin C: Results of a Randomized Phase III Trial. Journal of the National Cancer Institute, 93, 597-604. https://doi.org/10.1093/jnci/93.8.597

[32] Zhang, H., Wang, C., Chen, B. and Wang, X. (2012) Daunorubicin-TiO 2 Nanocomposites as a "Smart" pH-Responsive Drug Delivery System. International Journal of Nanomedicine, 7, 235-242.

[33] Gan, Q. and Wang, T. (2007) Chitosan Nanoparticle as Protein Delivery CarrierSystematic Examination of Fabrication Conditions for Efficient Loading and Release. Colloids and Surfaces B Biointerfaces, 59, 24-34.

[34] Nishiyama, M., Suzuki, K. and Kumazaki, T. (1997) Molecular Targeting of Mitomycin C Chemotherapy. International Journal of Cancer, 72, 649-665. https://doi.org/10.1002/(SICI)1097-0215(19970807)72:4<649::AID-IJC17>3.0.CO;2$\underline{6}$

[35] Crooke, S.T. and Bradner, W.T. (1976) Mitomycin C: A Review. Cancer Treatment Reviews, 3, 121-139.

[36] Ko, J.C., Chen, J.C., Wang, T.J., Zheng, H.Y., Chen, W.C, Chang, P.Y. and Lin, Y.W. (2016) Astaxanthin Down-Regulates Rad51 Expression via Inactivation of AKT Kinase to Enhance Mitomycin C-Induced Cytotoxicity in Human Non-Small Cell Lung Cancer Cells. Biochemical Pharmacology, 105, 91-100.

[37] Deng, X., Cao, M., Zhang, J., Hu, K., Yin, Z., Zhou, Z., Xiao, X., Yang, Y., Sheng, W., Wu, Y. and Zeng, Y. (2014) Hyaluronic Acid Chitosan Nanoparticles for Co-Delivery of MiR-34a and Doxorubicin in Therapy against Triple Negative Breast Cancer. Biomaterials, 35, 4333-4344.

[38] Kini, D.B. and Panda, D. (2015) Mechanism of Anti-Cancer Activity of Benomyl Loaded Nanoparticles in Multidrug Resistant Cancer Cells. Journal of Biomedical Nanotechnology, 11, 877-889. https://doi.org/10.1166/jbn.2015.1998 